\title{
One-year follow-up after sacrospinous hysteropexy and vaginal hysterectomy for uterine descent: a randomized study
}

\author{
Viviane Dietz • Carl H. van der Vaart . \\ Yolanda van der Graaf • Peter Heintz • \\ Steven E. Schraffordt Koops
}

Received: 17 March 2009 / Accepted: 23 September 2009/Published online: 16 October 2009

(C) The Author(s) 2009. This article is published with open access at Springerlink.com

\begin{abstract}
Introduction and hypothesis In a retrospective study, the sacrospinous hysteropexy was associated with a shorter recovery time compared to a vaginal hysterectomy with no differences in anatomical outcomes. No randomized trials are performed.

Methods Sixty-six women with stage $2-4$ uterine descent were randomized for vaginal hysterectomy(31) or sacrospinous hysteropexy(35). Recovery time, anatomical outcomes, functional outcomes, and quality of life were measured.

Results Length of time to return to work was shorter after a sacrospinous hysteropexy (43 versus 66 days, $p=0.02$ ). The difference in risk for recurrent prolapse stage 2 or more of the apical compartment at 1-year follow-up was $17 \%(95 \%$ confidence interval, 2 to 30 ) in favor of the vaginal
\end{abstract}

\footnotetext{
V. Dietz $(\bowtie)$

Department of Obstetrics and Gynecology,

Catharina Medical Center,

Michelangelolaan 2,

5623 Eindhoven, The Netherlands

e-mail: viviane.dietz@sapio.nl

C. H. van der Vaart $\cdot$ P. Heintz

Department of Perinatology and Gynecology,

University Medical Center Utrecht,

Utrecht, The Netherlands

Y. van der Graaf

Julius Center of Health Science and Primary Care,

University Medical Center Utrecht,

Utrecht, The Netherlands

S. E. Schraffordt Koops

Department of Obstetrics and Gynecology,

Meander Medical Center,

Amersfoort, The Netherlands
}

hysterectomy. No differences in quality of life and urogenital symptoms were found.

Conclusions The sacrospinous hysteropexy for uterine descent is associated with an earlier recovery time, more recurrent apical prolapses but no differences in functional outcomes, and quality of life.

Keywords Anatomical outcomes · Pelvic organ prolapse · Quality of life · Recovery time · Sacrospinous hysteropexy · Vaginal hysterectomy

\section{Introduction}

Pelvic organ prolapse is a major health problem that will increase in the next decennia due to increased life expectancy. In 1989, Richardson reported on the sacrospinous hysteropexy, a procedure performed in case of a uterine descent, in which the uterus could be preserved [1]. Today, it is unclear whether removing the uterus is necessary or leads to better results. In several studies, it has been shown that sacrospinous hysteropexy is anatomically efficient and safe, and most women have been highly satisfied with the procedure [1-6]. In only three nonrandomized studies, the sacrospinous hysteropexy was compared with a vaginal hysterectomy in terms of anatomical outcomes [4-6]. The procedures were equally effective with regard to anatomical outcomes. Recovery time after a sacrospinous hysteropexy has been shown to be significantly shorter compared to vaginal hysterectomy in a retrospective study [4]. The only randomized study ever performed involving sacrospinous hysteropexy and vaginal hysterectomy focused solely on sexual function 6 months after surgery [7]. 
A recent editorial comment in this journal concluded that "no proper randomized trial of hysterectomy versus nonhysterectomy in uterine prolapse repair has yet been done (and certainly, will not be easy to do") [8].

We performed a randomized study comparing the vaginal hysterectomy with the sacrospinous hysteropexy for the treatment of uterine descent stage 2-4 in women with no medical history of pelvic surgery and with a normal uterus, cervix, and ovaries and compared differences in recovery time. Also, data on recurrent prolapse stage2-4, recurrent prolapse surgery, functional outcomes, and quality of life at 1-year follow-up are presented.

\section{Materials and methods}

This nonblinded, randomized study was approved by the ethics committees of the six participating hospitals (University Center Utrecht, Meander Medical Center Amersfoort, Rijnstate Hospital Arnhem, St. Antonius Hospital Nieuwegein, St. Elisabeth Hospital Tilburg, and Twee Steden Hospital Tilburg).

\section{Patients}

Women were referred to one of the hospitals by their general practitioner because of symptomatic pelvic organ prolapse. Between February 1, 2004 and December 1, 2006, women were invited to participate. Criteria for entry into the study were the presence of a uterine descent stage 2-4 according to the classification of the International Continence Society and the patient's desire for surgical correction [9]. Other selection criteria were no medical history of pelvic surgery, normal uterus and ovaries on ultrasound examination, normal menstrual bleeding pattern (if premenopausal), normal cervical cytology, and no insulin-dependent diabetes mellitus. After informed consent, patients were randomly assigned by drawing sealed and opaque envelopes for one of the two types of prolapse surgery. Randomization was centralized. The gynecologist contacted the research nurse or the principal investigator when randomization was needed. The gynecologist was informed about the surgical procedure by phone and mail.

\section{Measurements}

Primary outcome of the study was recovery time after surgery including return to daily activities and resumption of work activities. Secondary outcomes were complications, anatomical outcomes, functional outcomes, and quality of life at 1-year follow-up.
Baseline characteristics were collected. All women were asked to complete a diary considering recovery time and when applicable, returning to work.

Postoperative pain management in each hospital was done according to the protocol. Information about complications during and after surgery and length of hospital stay were collected in the clinical research file of all study participants. All women were counseled the same about restrictions at home and at work. No heavy lifting, heavy house chores, exercise, and work was allowed in the first 6 weeks. At baseline and 1 year after surgery, women underwent pelvic examination using the pelvic organ quantification system (POP-Q) [9]. We compared the recurrence rate stage $2-4$ of the apical, anterior, and posterior compartment after a vaginal hysterectomy and sacrospinous hysteropexy. A prolapse stage 2-4 on gynecological examination 1 year after surgery was considered a recurrence. The necessity for repeat surgery was indicated by the treating gynecologist. Functional outcomes and quality of life were measured with the urinary distress inventory (UDI) and the incontinence impact questionnaire (IIQ) before surgery and 1 year after surgery, which was translated and validated for the Dutch population [10]. The scores of these domains vary between zero and 100. A high score on a particular domain indicates more bothersome symptoms or the worst quality of life.

\section{Surgical procedures}

Experienced gynecologists from the six participating hospitals performed all vaginal hysterectomy procedures. Sacrospinous hysteropexy procedures were performed by experienced gynecologists with special skills in pelvic floor surgery who had performed at least 20 sacrospinous hysteropexy procedures before the start of the study. The vaginal hysterectomy and sacrospinous hysteropexy were combined with an anterior or posterior colporrhaphy (fascia plication) or both with absorbable Vicryl 2-0 interrupted sutures (Ethicon, Sommerville, New Jersey, USA) when indicated by the judgment of the individual gynecologist. In the vaginal hysterectomy group, the uterosacral ligaments were reattached with resorbable sutures (Vicryl 1, Ethicon, Sommerville, New Jersey, USA) to the vaginal cuff after removal of the uterus [11].

The sacrospinous hysteropexy was performed unilaterally to the right ligament. A midline incision in the posterior vaginal wall was extended to the posterior part of the cervix. In two women, a transverse incision was made $2 \mathrm{~cm}$ below the fornix posterior because of the preference of the surgeon. Through sharp and blunt dissection, the right sacrospinous ligament was made visible with the use of three Breisky retractors. Two 
nonabsorbable Prolene 1 sutures (Ethicon, Sommerville, New Jersey, USA) were placed through the right sacrospinous ligament, approximately $2 \mathrm{~cm}$ medial to the ischial spine, and subsequently, placed through the posterior side of the cervix in the midline. The cervix was placed in close contact with the ligament without a suture bridge. In case of coexisting urinary stress incontinence, a surgical procedure using tension-free vaginal tape (Ethicon, Sommerville, New Jersey, USA) was performed retropubic as described by Ulmsten.[12]. All women received perioperative thrombosis prophylaxis and a single dose of intravenous prophylactic antibiotic before surgery. Postoperatively, an indwelling bladder catheter was placed in all women and removed $24 \mathrm{~h}$ after surgery (except after an additional anterior colporrhaphy, in which case it was left in place for 3 days).

\section{Sample size calculation}

The sample size was calculated for the primary outcome measurement, recovery time. With an expected difference between groups of $25 \%$ (68\% fully recovered within 3 months after a sacrospinous hysteropexy and $43 \%$ after a vaginal hysterectomy in a previous retrospective study) [4], a power of $80 \%$, and an alpha of 0.05 ; at least 61 women in each group were needed to be enrolled.

\section{Statistical analysis}

An intention to treat analysis was performed to calculate which of the two surgical procedures scored best with respect to the outcome measurements and to calculate the difference in risk and corresponding 95\% confidence intervals of recurrent prolapse. Descriptive statistics were used for continuous (means and standard deviation) and categorical (numbers/percentages) data. Differences between the groups were analyzed with an unpaired Student's $t$ test for continuous variables and the chi-square test for categorized variables. Before and after surgery, domain scores on functional outcomes and quality of life were calculated with a paired Student's $t$ test. A regression analysis was performed to correct age. A $p$ value of $<0.05$ was considered significant.

To handle the problem of missing data on POP-Q stage, we calculated two different scenarios. First, we used data on POP-Q stage of the last gynecological examination available, known as the last observation carried forward method [13]. Second, we used the worst-case scenario method in which all women who were not seen for gynecological examination 1 year after surgery were regarded as having a recurrent prolapse stage 4 on all compartments. All statistical analyses were performed using confidence interval analysis and SPSS [14].

\section{Results}

Seventy-one women with symptomatic uterine descent stage 2-4 requiring surgery were randomized between February 2004 and December 2006. A trial profile of subject enrollment is displayed in Fig. 1. After randomization and before surgery, five women withdrew from further participation. In three of these five women, surgery was cancelled because the women stated that their complaints decreased. One woman was diagnosed with atypical endometrial hyperplasia after a diagnostic workup and therefore, was treated differently. One woman refused to participate because answering the questionnaires was regarded as too much of a bother. In the remaining 66 women, there were six protocol deviations; two women underwent a vaginal hysterectomy instead of a sacrospinous hysteropexy (one woman because of inadequate visualization of the sacrospinous ligament, and in one woman, the reason for changing the procedure was not written down adequately in the clinical research file); and four women had an exclusion criterion that was overlooked before randomization. Two of them were diagnosed with insulin-dependent diabetes mellitus (one in each group) and two of them had a medical history of pelvic surgery (one in each group, cesarean delivery, and posterior colporrhaphy).

Not all women returned the diary and/or questionnaires (see Fig. 1) for a variety of reasons but mostly because this was too much of a bother. All these women, except for one, were contacted at 1-year follow-up and reported no symptoms except for recurrent stress incontinence $(n=1)$.

Two women of the sacrospinous hysteropexy group did not return for a gynecological examination at 1-year followup. One woman was lost to follow-up 6 weeks after surgery for unknown reasons. The other woman was only examined at 6-month follow-up. None of these women had returned to their hospital or general practitioner because of recurrent prolapse symptoms at 1-year follow-up.

Table 1 shows the baseline characteristics of the 71 randomized women and the surgical procedures performed in the 66 analyzed women. The baseline table shows no incomparability in the patient characteristics in the two groups, except age. The mean age of women who underwent a vaginal hysterectomy was about 2 years higher than the mean age of women who underwent a sacrospinous hysteropexy.

In both groups, no bladder or rectal injuries occurred; no blood transfusions or intensive care admissions were needed. Five days after surgery, one woman who underwent a vaginal hysterectomy was diagnosed with a distal occlusion of the right ureter. A nephrostomy catheter was placed that relieved the pain symptoms, and the patient was treated with antibiotics. At a 4-month follow-up, 
Fig. 1 Trial profile

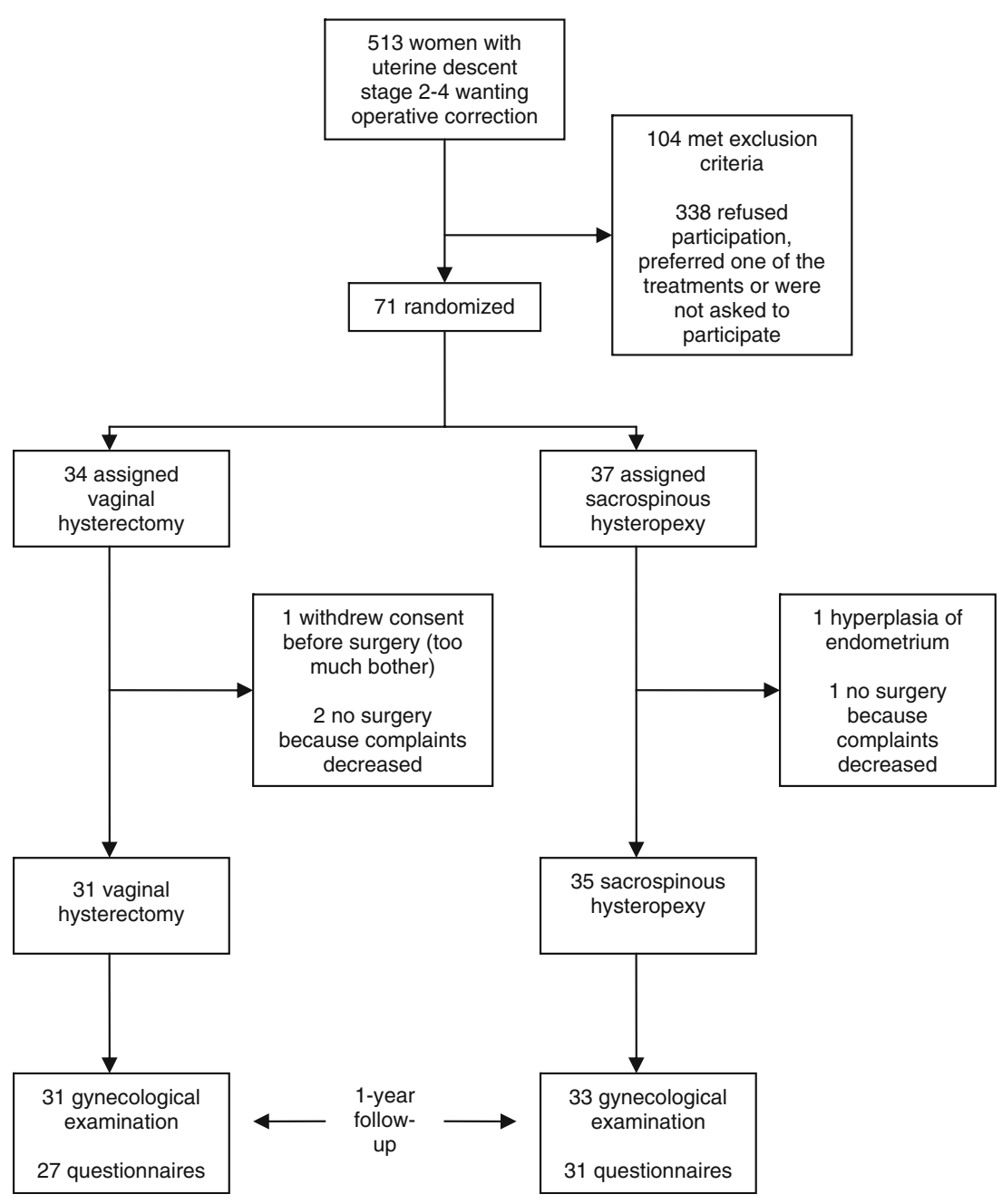

normal ureter function returned, and the patient recovered completely.

In Table 2, hospital stay and time returning to working activities are shown. Women returned to work 23 days earlier after a sacrospinous hysteropexy compared with women after a vaginal hysterectomy.

In Table 3, the mean total vaginal length (TVL) and point $\mathrm{C}, \mathrm{D}, \mathrm{Ba}$, and $\mathrm{Bp}$ of the $\mathrm{POP}-\mathrm{Q}$ in both groups is summarized. Women who underwent a sacrospinous hysteropexy had a higher mean TVL 1 year after surgery. Also, point D was significantly higher. In some women, only POP-Q stage was written down in the clinical research file. This explains the number of missing data in Table 3 .

In Table 4 , the stages of pelvic organ prolapse 1 year after surgery are shown. The data at 1-year follow-up include the anatomical outcome of three women after a sacrospinous hysteropexy who already underwent repeat surgery because of recurrent prolapse (two of these three because of apical prolapse). This means that at 1-year follow-up, $7+2$ women $(9 / 34,27 \%)$ had recurrent uterine descent stage 2 or more. Three women who underwent a sacrospinous hysteropexy had a stage 4 prolapse before surgery. All these women were diagnosed with a recurrent uterine descent within 1 year. Two of these women underwent repeat surgery.

Repeat surgery for prolapse after a sacrospinous hysteropexy was performed in 11\% (4/35) of patients (vaginal hysterectomy and/or colporrhaphy anterior). Surgery for prolapse after a vaginal hysterectomy occurred in $7 \%(2 / 31)$ of patients (colporrhaphy anterior and posterior; risk difference, $5 \%$; 95\% CI=-9 to 19$)$.

In Table 5, domain scores (scale from 0 to 100) of the UDI and IIQ are shown before and 1 year after surgery, according to surgical approach. No statistical differences in domain scores were found between the surgical procedures before surgery and 1 year after surgery. Both procedures showed an improvement in symptoms in all domains of the UDI and IIQ at 1-year follow-up. These improvements were statistically significant except for the obstructive micturition domain, which was of borderline significance $(p=0.06)$ in the sacrospinous hysteropexy group. In the vaginal hysterectomy group, there was no statistically 
Table 1 Baseline characteristics of the women at randomization, POP-Q, and surgical procedures performed

\begin{tabular}{|c|c|c|}
\hline & $\begin{array}{l}\text { Sacrospinous } \\
\text { hysteropexy } \\
(n=37)\end{array}$ & $\begin{array}{l}\text { Vaginal } \\
\text { hysterectomy } \\
(n=34)\end{array}$ \\
\hline Age (mean \pm SD) & $61.5 \pm 9.6$ & $63.7 \pm 9.0$ \\
\hline $\begin{array}{l}\text { Body mass index } \\
(\text { mean } \pm \mathrm{SD})\end{array}$ & $26.3 \pm 3.2$ & $25.9 \pm 2.9$ \\
\hline Parity [median (range)] & $2[0-5]$ & $2[1-7]$ \\
\hline Postmenopausal & $33(89)$ & $32(94)$ \\
\hline \multicolumn{3}{|l|}{ Prior surgery } \\
\hline Appendectomy & $4(11)$ & $5(15)$ \\
\hline Sterilization & $4(11)$ & $4(12)$ \\
\hline $\begin{array}{l}\text { Hemicolectomy (colon } \\
\text { carcinoma) }\end{array}$ & $1(3)$ & $0(0)$ \\
\hline \multicolumn{3}{|l|}{ Relevant comorbidity } \\
\hline $\begin{array}{l}\text { Insulin-dependent diabetes } \\
\text { mellitus }\end{array}$ & $1(3)$ & $1(3)$ \\
\hline $\begin{array}{l}\text { Noninsulin-dependent } \\
\text { diabetes mellitus }\end{array}$ & $1(3)$ & $2(6)$ \\
\hline $\begin{array}{l}\text { Chronic obstructive } \\
\text { pulmonary disease }\end{array}$ & $3(8)$ & $3(9)$ \\
\hline Rheumatoid arthritis & $0(0)$ & $2(6)$ \\
\hline \multicolumn{3}{|c|}{ Stage of prolapse before surgery (POP-Q) } \\
\hline Uterine descent: stage $2-4$ & $37(100)$ & $34(100)$ \\
\hline Cystocele: stage $0-1$ & $3(8)$ & $2(6)$ \\
\hline Stage $2-4$ & $34(92)$ & $32(94)$ \\
\hline Rectocele: stage $0-1$ & $14(38)$ & $18(53)$ \\
\hline Stage $2-4$ & $23(62)$ & $16(47)$ \\
\hline $\begin{array}{l}\text { Surgical procedures } \\
\text { performed }\end{array}$ & $(n=35)$ & $(n=31)$ \\
\hline Sacrospinous hysteropexy & $33(94)$ & $0(0)$ \\
\hline Vaginal hysterectomy & $2(6)$ & $31(100)$ \\
\hline Anterior colporrhaphy & $28(80)$ & $31(100)$ \\
\hline Posterior colporrhaphy & $20(57)$ & $15(48)$ \\
\hline Tension-free vaginal tape & $4(11)$ & $3(10)$ \\
\hline
\end{tabular}

Data are presented as $n(\%)$ unless otherwise noted

$S D$ standard deviation

significant improvement in the urinary incontinence domain $(p=0.2)$.

Adjusting for age did not alter our findings as described before. Also, analyzing the outcome measurements per protocol did not influence the results outlined except for the differences in median hospital stay ( 3 versus 4 days, $p=$ 0.09 in favor of the sacrospinous hysteropexy).

\section{Discussion}

This multicenter, randomized study showed that the median length of hospital stay and the length of time to return to work were significantly shorter after a sacrospinous hysteropexy as compared to the vaginal hysterectomy. The two procedures are comparable with respect to complication rate and functional outcomes and quality of life at 1-year follow-up. Women who underwent a vaginal hysterectomy for uterine descent stage 2 or more had considerably fewer recurrences $(3 \%)$ of the apical compartment compared to women after a sacrospinous hysteropexy (27\%). Especially high recurrence was noted in the preoperative high grade prolapse patients who underwent a sacrospinous hysteropexy.

Significant differences were found between the two groups with regard to the resumption of work activities. In a retrospective study comparing a vaginal hysterectomy with a sacrospinous hysteropexy, women were asked about the length of time to complete recovery after surgery [4]. After a vaginal hysterectomy, $57 \%$ of women needed more than 3 months to recover compared with $32 \%$ of women after a sacrospinous hysteropexy, which was a statistically significant difference. In our study, both groups needed almost 34 days to resume daily activities, which was also reported by Maher et al. [5]. No other studies comparing the vaginal hysterectomy and sacrospinous hysteropexy were available with data on recovery time. When women were specifically asked about returning to work activities, the women in the vaginal hysterectomy group needed significantly more time ( 23 days). The differences in surgical techniques could be responsible for a shorter recovery time after a sacrospinous hysteropexy. We did not ask why women after a vaginal hysterectomy needed more time to recover and go back to work, and we also did not ask about the type of work. This can be regarded as a shortcoming of the study. It appears that the differences in recovery time are also reflected in the median length of hospital stay, which was 1 day shorter after a sacrospinous hysteropexy. Aside from the differences in surgical techniques, other factors also have to be considered. It is

Table 2 Hospital stay and recovery time according to surgical approach

\begin{tabular}{|c|c|c|c|}
\hline & $\begin{array}{l}\text { Sacrospinous } \\
\text { hysteropexy } \\
(n=35)\end{array}$ & $\begin{array}{l}\text { Vaginal } \\
\text { hysterectomy } \\
(n=31)\end{array}$ & $\begin{array}{l}p \\
\text { value }\end{array}$ \\
\hline \multicolumn{4}{|c|}{ Hospital admission (days): } \\
\hline Median (range) & $3(3-7)$ & $4(3-14)$ & $0.03^{\mathrm{a}}$ \\
\hline Recovery time (days) & $(n=29)$ & $(n=28)$ & \\
\hline $\begin{array}{l}\text { - Return to daily } \\
\text { activities }\end{array}$ & $34(13)$ & $33(21)$ & 0.9 \\
\hline $\begin{array}{l}\text { - Return to working } \\
\text { activities }\end{array}$ & $43(21)$ & $66(34)$ & 0.02 \\
\hline $\begin{array}{l}\text { - Completely return to } \\
\text { work }\end{array}$ & $57(33)$ & $80(45)$ & 0.08 \\
\hline
\end{tabular}

Data are means (standard deviation)

$p$ values calculated with independent Student's $t$ test

${ }^{a} p$ value was calculated with Mann-Whitney $U$ test 
Table 3 Pelvic organ prolapse quantification system: point C, D, Ba, $\mathrm{Bp}$, and TVL before surgery and 12 months after surgery

\begin{tabular}{|c|c|c|c|c|}
\hline & $\begin{array}{l}\text { Sacrospinous } \\
\text { hysteropexy } \\
(n=30)\end{array}$ & $\begin{array}{l}\text { Vaginal } \\
\text { hysterectomy } \\
(n=27)\end{array}$ & $\begin{array}{l}\text { Difference } \\
(95 \% \mathrm{CI})\end{array}$ & $\begin{array}{l}p \\
\text { value }\end{array}$ \\
\hline $\begin{array}{l}\text { Before } \\
\text { surgery }\end{array}$ & $n(\mathrm{SD})$ & $n(\mathrm{SD})$ & & \\
\hline - TVL & $8.1(0.9)$ & $7.8(1.0)$ & & \\
\hline$\cdot \mathrm{C}$ & $1.5(2.1)$ & $1.6(2.8)$ & & \\
\hline$\cdot \mathrm{D}$ & $-1.3(4.2)$ & $-0.7(5.3)$ & & \\
\hline - $\mathrm{Ba}$ & $1.8(2.3)$ & $2.2(2.1)$ & & \\
\hline - $\mathrm{Bp}$ & $-1.0(2.1)$ & $-0.1(3.5)$ & & \\
\hline $\begin{array}{l}1 \text { year after } \\
\text { surgery }\end{array}$ & $(n=30)$ & $(n=29)$ & & \\
\hline - TVL & $8.8(1.3)$ & $7.3(1.5)$ & $\begin{array}{l}1.5(0.7- \\
2.2)\end{array}$ & $<0.01$ \\
\hline$\cdot \mathrm{C}$ & $-5.1(3.6)$ & NA & NA & NA \\
\hline$\cdot \mathrm{D}$ & $-7.4(2.6)$ & $-5.7(1.9)$ & $\begin{array}{l}-1.7(-2.9- \\
-4.4)\end{array}$ & 0.01 \\
\hline - $\mathrm{Ba}$ & $-1.1(1.9)$ & $-0.7(1.5)$ & $\begin{array}{l}-0.5(-1.3- \\
0.4)\end{array}$ & 0.4 \\
\hline - $\mathrm{Bp}$ & $-2.2(1.2)$ & $-2.0(1.3)$ & $\begin{array}{l}-0.2(-0.9- \\
0.4)\end{array}$ & 0.5 \\
\hline
\end{tabular}

Data are numbers (standard deviation)

$N A$ not available

possible that the doctor released women earlier after a sacrospinous hysteropexy because this procedure was considered less complex. Also, women might have felt that after a sacrospinous hysteropexy, recovery time would be shorter because surgery appeared less invasive to them. However, all women were counseled the same way about recovery time after surgery; so, we do not think this had a major influence on the outcome. Although this study was not performed to evaluate the costs of these operations, the results could be of economic importance.

In the literature, only a few studies describe functional outcomes after a sacrospinous hysteropexy [2-6]. Validated questionnaires were used in three studies, all with a retrospective design [2-4]. In only one of these studies were preoperative functional outcomes described. Our domain scores were comparable to the domain scores in these studies. Roovers et al. also used validated questionnaires in a study comparing a vaginal hysterectomy for uterine descent with an abdominal approach [15]. The domain scores on urogenital symptoms and quality of life were comparable to our results after a vaginal hysterectomy. In all of these studies, a large improvement in prolapse symptoms was shown. The impact on urinary symptoms was smaller, but we can conclude that both procedures provided significant improvement in urogenital symptoms and quality of life. However, because the vaginal hysterectomy and the sacrospinous hysteropexy were performed concomitantly with an anterior colporrhaphy in (almost) all cases, we do not know which surgical procedure contributed the most to the improvement in urogenital symptoms.

Anatomical recurrences of the apical compartment after a sacrospinous hysteropexy are described in $0 \%$ to $15 \%$ of patients [16]. After a vaginal hysterectomy for uterine descent, recurrences of the apical compartment varied between $0 \%$ and $12 \%$ [16]. It should be noted that almost all of these studies were nonrandomized.

An explanation for the variety of recurrence rates in the literature is the heterogeneity of data collection. Most studies were retrospective and based on medical files, not on gynecological examinations performed and regardless of symptoms. The rate of recurrent surgery for apical prolapse was low in all studies $(0-7 \%$ for a vault prolapse and $0-5 \%$ for a recurrent uterine descent) [16]. Recurrences of the anterior, posterior, and apical compartment after a vaginal hysterectomy in our study were comparable to that of Roovers et al. [15]. Compared with nonrandomized prospective and retrospective studies, our recurrence rates

Table 4 Stage of prolapse 1 year after surgery according to surgical approach

\begin{tabular}{|c|c|c|c|c|c|}
\hline 1year after su & gery & & Vaginal & Difference & $p$ \\
\hline LOCF & & $n(\%)$ & $n(\%)$ & $\%$ & \\
\hline $\begin{array}{l}\text { Uterine } \\
\text { descent/ }\end{array}$ & $\begin{array}{l}\text { stage } \\
0-1\end{array}$ & $27(79)^{\mathrm{a}}$ & $30(97)$ & $17(2-32)$ & 0.03 \\
\hline $\begin{array}{l}\text { vaginal vault } \\
\text { descent }\end{array}$ & $\begin{array}{l}\text { stage } \\
2-4\end{array}$ & $7(21)$ & $1(3)$ & & \\
\hline Cystocele & $\begin{array}{l}\text { stage } \\
0-1\end{array}$ & $17(50)$ & $11(35)$ & $\begin{array}{l}-15 \\
(-38-9)\end{array}$ & 0.2 \\
\hline & $\begin{array}{l}\text { stage } \\
2-4\end{array}$ & $17(50)$ & $20(65)$ & & \\
\hline Rectocele & $\begin{array}{l}\text { stage } \\
0-1\end{array}$ & $28(82)$ & $22(71)$ & $\begin{array}{l}-11(-32- \\
9)\end{array}$ & 0.3 \\
\hline & $\begin{array}{l}\text { stage } \\
2-4\end{array}$ & $6(18)$ & $9(29)$ & & \\
\hline Worst-case sce & nario & $(n=35)$ & $(n=31)$ & & \\
\hline $\begin{array}{l}\text { Uterine } \\
\text { descent/ }\end{array}$ & $\begin{array}{l}\text { stage } \\
0-1\end{array}$ & $26(74)^{\mathrm{a}}$ & $30(97)$ & $23(7-38)$ & 0.01 \\
\hline $\begin{array}{l}\text { vaginal } \\
\text { vault } \\
\text { descent }\end{array}$ & $\begin{array}{l}\text { stage } \\
2-4\end{array}$ & $9(26)$ & $1(3)$ & & \\
\hline Cystocele & $\begin{array}{l}\text { stage } \\
0-1\end{array}$ & 17 (49) & $11(36)$ & $\begin{array}{l}-13 \\
(-37-11)\end{array}$ & 0.3 \\
\hline & $\begin{array}{l}\text { stage } \\
2-4\end{array}$ & $18(51)$ & $20(64)$ & & \\
\hline Rectocele & $\begin{array}{l}\text { stage } \\
0-1\end{array}$ & $27(77)$ & $22(71)$ & $\begin{array}{l}-6(-27- \\
15)\end{array}$ & 0.6 \\
\hline & $\begin{array}{l}\text { stage } \\
2-4\end{array}$ & $8(23)$ & $9(29)$ & & \\
\hline
\end{tabular}

LOCF last observation carried forward

${ }^{\mathrm{a}}$ In this group of women, two women had recurrent surgery for apical prolapse 
Table 5 Domain scores of the urinary distress inventory (UDI) and incontinence impact questionnaire (IIQ) before and after surgery according to surgical approach

\begin{tabular}{|c|c|c|c|c|c|c|}
\hline & \multicolumn{2}{|c|}{ Before surgery } & \multicolumn{4}{|c|}{ 1year after surgery } \\
\hline & $\mathrm{SSH}, n=34$ & $\mathrm{VH}, n=31$ & $\mathrm{SSH}, n=31$ & $\mathrm{VH}, n=27$ & Difference $(95 \% \mathrm{CI})$ & $p$ value \\
\hline \multicolumn{7}{|l|}{ UDI domain scores: } \\
\hline Overactive bladder & $22(20)$ & $25(24)$ & $11(18)$ & $12(17)$ & $0.5(-10.1-11.1)$ & 0.9 \\
\hline Urinary incontinence & $11(14)$ & $10(16)$ & $6(8)$ & $6(11)$ & $0.3(-4.8-5.4)$ & 1.0 \\
\hline Obstructive micturition & $11(19)$ & $23(26)$ & $3(9)$ & $8(16)$ & $-5.0(-13.6-3.6)$ & 0.1 \\
\hline Genital prolapse & $55(30)$ & $64(32)$ & $2(5)$ & $4(13)$ & $-2.5(-7.9-2.9)$ & 0.5 \\
\hline Pain & $22(19)$ & $24(25)$ & $11(18)$ & $7(21)$ & $2.5(-7.3-12.4)$ & 0.4 \\
\hline \multicolumn{7}{|l|}{ IIQ domain cores: } \\
\hline Physical functioning & $17(23)$ & $23(23)$ & $3(9)$ & $12(22)$ & $-9.9(-18.5--1.3)$ & 0.06 \\
\hline Mobility & $20(18)$ & $26(17)$ & $7(13)$ & $12(15)$ & $-3.6(-10.5-3.3)$ & 0.3 \\
\hline Emotional health & $13(15)$ & $12(19)$ & $5(8)$ & $6(11)$ & $-2.0(-6.9-3.0)$ & 0.5 \\
\hline Social functioning & $6(12)$ & $14(18)$ & $2(7)$ & $3(8)$ & $-0.9(-4.7-2.8)$ & 0.6 \\
\hline Embarrassment & $10(14)$ & $16(14)$ & $4(11)$ & $6(16)$ & $-2.6(-9.4-4.2)$ & 0.5 \\
\hline
\end{tabular}

Data are numbers (standard deviation)

SSH sacrospinous hysteropexy, $V H$ vaginal hysterectomy

UDI, $0=$ not bothersome and $100=$ most bothersome

IIQ, $0=$ best quality of life and $100=$ worst quality of life

of the apical compartment after the sacrospinous hysteropexy at 1-year follow-up were high. The number of women with and without a recurrent uterine descent was too small to make a statistical subanalysis. We did found that all women with a preoperative prolapse stage 4 had a recurrent apical prolapse after a sacrospinous hysteropexy. Two of these women underwent recurrent surgery. Lin et al. described a correlation between a high rate $(75 \%)$ of recurrent prolapse and a stage 3 or 4 prolapse preoperatively [17]. Based on their data, they advised against performing a sacrospinous hysteropexy in case of a stage 3 or 4 uterine descent. In our study, $44 \%$ of women who underwent a sacrospinous hysteropexy had a stage 3 or 4 uterine descent. This could have also influenced our recurrence rates. It could be that when the uterus is situated outside the vagina for a long time, the cervix becomes more fragile and erosive. This might be a risk factor for tearing the suture out of the cervix. In most reports on the sacrospinous hysteropexy, only a selected group of women (no stage 3 or 4 uterine descent) were included into the study $[5,6]$ Therefore, these studies are not comparable to our study. Recurrent uterine descent was already present at 6 months follow-up. After 6 weeks, women were instructed to expand their activities (but still not to perform heavy lifting). Possibly, after expanding activities, the Prolene sutures were torn from the cervix. Apical prolapse after a vaginal hysterectomy is, probably, based on stretching of the tissue (sacrouterine ligaments) over time and as a consequence, develop over time and therefore, were less found in our 1year follow-up. In the literature, it was stated that after a sacrospinous hysteropexy is performed, the vaginal axis would become more horizontal with a higher risk of developing a cystocele [18]. Smilen et al. could not confirm this hypothesis [19]. Although we agree that the vaginal axis will be different, we also did not find more cystoceles after a sacrospinous hysteropexy is compared with a vaginal hysterectomy at 1 -year follow-up.

The strength of our study was the randomization that made the two groups of women comparable. Thereby, in contrast to other comparative studies between the sacrospinous hysteropexy and the vaginal hysterectomy, we focused on recovery time, anatomical outcomes, functional outcomes and quality of life, and validated questionnaires were used [10].

We chose to perform a multicenter study to make the group of gynecologist who performed the surgery more generalizable compared with a 1-center study. This also can be of influence on the results of the outcomes because more different surgeons will also bring about more differences in experiences of the surgical techniques. We tried to avoid this by having a minimum of 20 procedures performed by the responsible gynecologist before the start of the study. Some limitations need to be addressed. The number of subjects fell short of the sample size that was calculated. As mentioned in the methods section, the sample size was calculated for postoperative recovery time, not for difference in functional outcomes. Unfortunately, many women were reluctant to let a lottery decide whether their uterus was to be removed. This is reflected in our study flow chart (Fig. 1). Only 17\% of the potential 409 women participated. A lot of women had a preference for one of the two 
procedures. These women could be divided into two groups. When women were told that at the time of the study we did not know whether one procedure was superior to the other, some chose to retain their uterus ("why remove the uterus if not necessary?"). Another group of women wanted to have their uterus removed because family members or friends had good experience with this procedure (first choice operation for decades). Thereby, some women think that when the uterus is removed, prolapse cannot occur anymore. Also, the fact that a removed uterus cannot give any health problems (cancer of the cervix or uterus) in future times could be a reason to prefer a vaginal hysterectomy. We do not think that the stage of prolapse or differences in symptoms was a reason for preferring one of the two procedures. Therefore, our results are most likely generalizable to all patients who meet the inclusion and exclusion criteria. If randomization were performed just before surgery or even in the operating room, fewer protocol violations would have occurred. However, we had chosen to randomize earlier to have more time to inform women about the procedure they were randomized to.

Based on the results of this study, a sacrospinous hysteropexy for the correction of uterine descent stage $2-4$ is associated with earlier return to work activities with no differences in quality of life and functional outcomes. Based on the anatomical results at 1-year follow-up, the vaginal hysterectomy had fewer recurrences in the whole group of high and low grade prolapse compared to the sacrospinous hysteropexy. A stage 4 prolapse preoperative was associated with a high recurrence rate of uterine descent after a sacrospinous hysteropexy with the need for recurrent surgery. Longer follow-up data will follow. A large cohort study will be needed to search for risk factors for having anatomical recurrent prolapse after a sacrospinous hysteropexy.

Acknowledgments We thank all the participating women and the following gynecologists for their cooperation: C.I.M. Aalders, E. van Beek, E. Bolkzijl, M.J. Duk, J.F. ter Haar, M.M. IJland, E. Lenters, P.C.M. van de Salm, J.H. Schagen van Leeuwen, W.A. Schöls, H.S. The, H.A.M. Vervest, W. van Vliet, M.C. Vos (Meander Medical Center Amersfoort, St. Antonius Hospital Nieuwegein, St. Elisabeth Hospital Tilburg, Rijnstate Hospital Arnhem, and Twee Steden Ziekenhuis Tilburg).

\section{Conflicts of interest None.}

Open Access This article is distributed under the terms of the Creative Commons Attribution Noncommercial License which permits any noncommercial use, distribution, and reproduction in any medium, provided the original author(s) and source are credited.

\section{References}

1. Richardson DA, Scotti RJ, Ostergard DR (1989) Surgical management of uterine prolapse in young women. J Reprod Med 34(6):388-392
2. Dietz V, de Jong J, Huisman M, Schraffordt Koops S, Heintz P, van der Vaart H (2007) The effectiveness of the sacrospinous hysteropexy for the treatment of uterovaginal prolapse. Int Urogynecol J 18:1271-1276

3. Dietz V, Huisman M, de Jong JM, Heintz PM, van der Vaart CH (2008) Functional outcome after sacrospinous hysteropexy for uterine descensus. Int Urogynecol J 19(6): $747-752$

4. Brummen HJ, van de Pol G, Aalders CIM, Heintz APM, van der Vaart CH (2003) Sacrospinous hysteropexy compared to vaginal hysterectomy as primary surgical treatment for a descensus uteri: effects on urinary symptoms. Int Urogynecol J 14:350-355

5. Maher CF, Cary MP, Slack CJ, Murray CJ, Milligan M, Schluter P (2001) Uterine preservation or hysterectomy at sacrospinous colpopexy for uterovaginal prolapse. Int Urogynecol J 12:381385

6. Hefni M, El-Toukhy T, Bhaumik J, Katsimanis E (2003) Sacrospinous cervicocolpopexy with uterine conservation for uterovaginal prolapse in elderly women: an evolving concept. Am J Obstet Gynecol 188(3):645-650

7. Jeng CJ, Yang YC, Tzeng CR, Shen J, Wang LR (2005) Sexual functioning after vaginal hysterectomy or transvaginal sacrospinous uterine suspension for uterine prolapse. J Reprod Med 50:669-674

8. Walters MD (2008) Uterovaginal prolapse in women desiring uterine preservation. Int Urogynecol J 19(11): 1465-1470

9. Bump RC, Mattiasson A, Bo K et al (1996) The standardization of terminology of female pelvic organ prolapse and pelvic floor dysfunction. Am J Obstet Gynecol 175:10-17

10. van der Vaart CH, de Leeuw JR, Roovers JP, Heintz AP (2003) Measuring health-related quality of life in women with urogenital dysfunction: the urogenital distress inventory and incontinence impact questionnaire revisited. Neurourol Urodyn 22:97-104

11. Hirsch HA, Kaser O, Iklé FA (1997) Atlas of Gynecologic Surgery. Thieme-Stuttgard, New York, pp 226-238

12. Ulmsten U, Hendriksson L, Johnson P, Vahros G (1996) An ambulatory surgical procedure under local anesthesia for treatment of female urinary incontinence. Int Urogynecol $\mathrm{J} 7$ (2):81-85

13. Mazumdar S, Liu KS, Houck PR, Reynolds CF (1999) Intentto-treat analysis for longitudinal clinical trials: coping with the challenge of missing values. J Psychiatric Research 33:87-95

14. Altman DG, David Machin D, Trevor N Bryant TN, Gardner MJ (2000) Statistics with Confidence. BMJ Books 2nd Edition, London, ISBN: 0727913751

15. Roovers JP, van der Vaart CH, van der Bom JG, van Leeuwen JH, Scholten PC, Heintz AP (2004) A randomized controlled trial comparing abdominal and vaginal prolapse surgery: effects on urogenital function. BJOG 111:50-56

16. Schraffordt DV, Koops SE, van der Vaart CH (2008) Vaginal surgery for uterine descent: which options do we have? A literature review. Int Urogynecol J 20(3):349-356

17. Lin TY, Su TH, Wang YL, Lee MY, Hsieh CH, Wang KG, Chen GD (2005) Risk factors for failure of transvaginal sacrospinous uterine suspension in the treatment of uterovaginal prolapse. J Formos Med Assoc 4:249-253

18. Carey MP, Slack MC (1994) Transvaginal sacrospinous colpopexy for vault and marked uterovaginal prolapse. $\mathrm{Br} \mathrm{J}$ Obstet Gynecol 101:536-540

19. Smilen SW, Saine J, Wallach SJ, Porges RF (1998) The risk of cystocele after sacrospinous ligament fixation. Am J Obstet Gynecol 179:1465-1471 